\title{
Proclus dans la première quaestio collativa de Gilles Charlier
}

\author{
Zénon Kaluza \\ Centre national de la recherche scientifique, PSL, LEM - UMR 8584, Paris
}

Ayant eu, vers la fin des années 60 et au début des années 70 du siècle passé, l'occasion de consulter assez régulièrement les manuscrits de Gilles Charlier, ceux de sa bibliothèque et ceux contenant ses écrits, dont le Commentaire des Sentences, je me suis alors persuadé de la forte présence de Proclus dans son Commentaire: non pas massive, certes, mais constante. Répondant donc à l' invitation des amis pour présenter plus en détail ce Commentaire, j' ai organisé mon exposé en deux parties. Dans la première, je présente rapidement la présence du théologien parisien dans la recherche historique et son commentaire; dans la seconde je montre la présence de l'Elementatio theologica de Proclus dans la quaestio collativa du premier Principium du Commentaire.

Gilles Charlier et son époque, son Commentaire des Sentences et la première quaestio collativa

Pourtant j'ai eu encore une deuxième raison, gardée secrète, pour retourner à Gilles Charlier et à ce moment historique exceptionnel, propice à la rencontre de quelques hommes et de quelques idées que le chancelier Jean Gerson s' est efforcé de faire disparaître de la Faculté de théologie sinon de l'Université. Toutefois, au début de février 1415, Gerson, principal pourfendeur de toute forme du platonisme, a quitté Paris pour se rendre au concile de Constance, sans savoir qu' il quitte Paris définitivement ${ }^{1}$. Quittant sa Faculté modérément affligée, il a sans doute laissé aux jeunes théologiens un peu de liberté et la scène de théologie universitaire peuplée par de grandes figures du passé plus

1 Glorieux 196oa, p. 128. Pour Gerson donnant quelques directives aux Messieurs de Navarre, voir ses Épitres $\mathrm{n}^{\circ}$ 4-7, p. 29-43; et pour son antiplatonisme, voir ses Duae lectiones contra vanam curiositatem, p. 224-249; Kaluza 1988, Annexe 1, p. 128-144. 
ou moins lointain, comme celles du Ps.-Denys l' Aréopagite, d'Albert le Grand et de Thomas d'Aquin, par deux philosophes grecs - Aristote et Proclus, et au moins trois penseurs musulmans - Avicenne, Alfarabi et Averroès et un juif Raby Moys, soit Moïse Maimonide. Ils seront tous là, dans les têtes et dans les textes de jeunes théologiens enseignants et contestateurs en cette année académique 1416-1417, quand, dans son collège de Cambrai, Gilles Charlier donnait ses leçons sur les Sentences ${ }^{2}$. L'alignement de tels noms laisse supposer qu'Albert le Grand n' est pas loin. La salle réunissait tous les docteurs, bacheliers et étudiants de la Faculté pour assister et entendre chaque année les Principia, ces ouvertures solennelles des cours sur chacun des quatre livres des Sentences ${ }^{3}$. Alors, qui, en 1416-1417, aurait pu participer, comme étudiant ou comme bachelier, dans les sessions solennelles des Principia? Et qui, à Paris, aurait pu manifester une ouverture sur les doctrines néoplatoniciennes, si fortement présentes déjà chez Albert le Grand ${ }^{4}$ ?

L'on sait qu'il y a tout d'abord eu à Paris un petit cercle de théologiens découverts par Gilles Gerard Meersseman et appelés «albertistes», à présent mieux connus grâce aux travaux récents ${ }^{5}$. Ces travaux jettent une lumière nouvelle sur le milieu intellectuel parisien, enrichissent nos informations et élargissent la perspective doctrinale qui manquaient gravement encore en $1995^{6}$.

Jean de Maisonneuve (de Nova Domo), étudiant en théologie, n' enseignait qu'à la Faculté des arts, où, au dire de son disciple Heimeric de Campo (van de

2 Swieżawski 1963; Swieżawski 1961 établit la date, contestée alors, des leçons sur les Sentences. Concernant la chronologie de la vie et des études de Charlier, voir Toussaint 1949; Gorochov 1997, p. 6o9-610; Sullivan 2011, p. 134-137. Par rapport à ces ouvrages, je repousse l' année de naissance de Gilles vers, ou même avant 1380, et argumente en faveur de ses études théologique au collège de Cambrai (contre le collège de Navarre); voir Kaluza 2015, surtout les notes 2 et 18 de l'introduction. J'y mentionne aussi les travaux récents. On s'imagine aisément Gilles Charlier quitter pour des raisons doctrinales le collège de Navarre, bien surveillé par Gerson depuis son retour de Bruges en septembre 1400; Glorieux 196ob, p. 113114 .

3 Glorieux 1969, surtout p. 138-141. L'année universitaire des théologiens s'ouvre le 14 septembre. A partir de ce jour on lit et discute un Principium par jour. Ces exercices doivent se terminer avant le 10 octobre. Les cours en théologie sont alors suspendus car les étudiants et les professeurs assistent aux Principia des sentenciaires. Cf. Kaluza 1987, p. 259-261, où je cite d' autres travaux; Zahnd 2016, p. 81-105 (84-87, Zur Textgattung der principia). Aux p. 8793 et 101-105, M. Ueli Zahnd discute et édite les mêmes gratiarum actiones de Gilles Charlier que j' ai appelées Supremum vale et publiées en 2015 (en réalité en 2016; cf. supra note 2). Il y note aussi l' existence d'une gratiarum actio de Lambert de Monte et de celle de Guillaume de Vorillon.

4 Libera 2005; Kaluza 1998.

5 Voir Meliadò, Negri 2011.

6 Cf. Kaluza 1995. 
Velde ou Aimeric de Campo), il a restauré la mémoire et les doctrines d'Albert le Grand. Brabançon comme son maître, Heimeric de Campo le dit primus Parisiensis doctrinae Alberti resuscitator ${ }^{7}$. Heimeric, maître ès arts à Paris à l'école de Maisonneuve, étudiait la théologie probablement depuis 1415 jusqu' en 1420. Dans les années où Charlier donnait ses leçons sur les Sentences, Heimeric était encore un tout jeune étudiant en théologie. À Cologne, puis à Louvain il a d'abord tenté de raviver l'opposition entre les thomistes et les albertistes pour ensuite essayer de les accorder. Il est important de noter que parmi ses premiers écrits on compte un Compendium divinorum, traité inspiré fortement par quelques auteurs néoplatoniciens, dont celui du Liber de causis ${ }^{8}$. Au concile de Bâle, Gilles Charlier retrouvera non seulement Heimeric, il y rencontrera un autre connaisseur de la philosophie de Proclus, Nicolas de Cues.

Nathalie Gorochov espérait nous faire «connaître [la] culture [des navarristes], leurs centres d'intérêt par la nature des livres qu'ils ont possédés». Peine pour une grande partie perdue, car, dit-elle encore: «La connaissance de ces bibliothèques d' anciens boursiers [du collège] est bien trop fragmentaire pour permettre une étude approfondie de leurs lectures ${ }^{9} »$. La liste de vingttrois boursiers ayant possédé ne serait ce qu' un seul manuscrit est, pour notre sujet, peu instructive. Il est cependant certain qu' aucune copie de l'Elementatio theologica de Proclus ne se trouvait parmi les livres de la riche bibliothèque de Pierre d'Ailly (70 mss.), de Jean de Courtecuisse (8o mss.), de Nicolas de Clamanges (20 mss.) et de Gilles Charlier $\left(25\right.$ mss.) qui l'utilise pourtant ${ }^{10}$. Gilles

$7 \quad$ Meersseman 1933, avec l'édition du De esse et essentia de Jean de Maisonneuve; Kaluza 1986; Kaluza 1988; Kaluza 1995, p. 210-214, 258. La recherche récente montre la présence, dans les écoles et les universités de l'Empire et de l'Europe centrale, d'un autre courant albertiste, jusqu' alors inconnu; cf. Calma 2016.

8 Heymeric de Campo, Compendium divinorum. Ses écrits commencent à paraître, voir, par exemple, cinq courts écrits dans Heymericus de Campo, Opera selecta; Calma, Imbach 2008 et 2013, étudient et éditent le traité métaphysique Colliget principiorum. Ce qui reste des leçons sur les Sentences est publié par Hoenen 2002. Moi même, j' ai publié un court commentaire doctrinal d'Heimeric sur l'Alphabetum, première partie de son Testamentum doctrinale: Kaluza 1992.

9 Gorochov 1997, p. 502. Sur ce point, il est sans importance que Charlier soit logé dans le voisin collège de Cambrai: les comparaisons restent valides. Voir aussi pour le même milieu une contribution de Gilbert Ouy 1989.

10 Ouy 1989, p. 503, 6o9. Pour la bibliothèque de Gilles Charlier, laquelle jadis m’ intéressait beaucoup et dont je me suis occupé de près. Pierre d'Ailly ne se réfère pas à Proclus dans ses Principia aux Sentences. Chappuis 1993, p. 95, suppose une «interprétation néoplatonicienne» d' un passage de la Métaphysique d'Aristote (dans le texte de la p. $158^{*}$, §3); et, p. 127, note 213, identifie la prop. 12 de l'Elementatio theologica de Proclus comme source de cette interprétation. Même si une belle page de Paul Vignaux, rappelée par Mme Chappuis 
Charlier aurait pu travailler sur un exemplaire emprunté à la bibliothèque de son collège, ou sur un manuscrit acheté et puis revendu. En soulevant à nouveau l'interrogation de Mme Gorochov, en limitant aussi sa portée à la seule œuvre de Gilles Charlier, nous allons jeter un coup d' œil rapide sur sa gratiarumactio afin de se reconnaître dans ses sources théologiques et de définir avec précision la position occupée par l'Elementatio theologica de Proclus.

Les théologiens faisant autorité sont énumérés et respectueusement loués dans cette pièce que Stefan Swieżawski appelle le discours de clôture et Ueli Zahnd die letzte Danksagung ou die Dankesrede. Il se lit dans le ms. 959 de la Bibliothèque Mazarine, aux f. $198^{\mathrm{va}}-199^{\mathrm{vb}}$, et dans le ms. Angers, Bibliothèque municipale, $205, \mathrm{f} .232^{\mathrm{r}}-233^{\mathrm{r}}$, donc à la fin du livre IV du Commentaire. Je répète ces noms célèbres, lesquels représentent ceux des tous les théologiens, dans l'ordre établi par Charlier: saint Paul, saint Augustin, Pierre Lombard, Thomas d'Aquin, Albert le Grand, Alexandre de Halès, Bonaventure, Durand de S. Pourçain, Gilles de Rome et Jean Duns $\operatorname{Scot}^{11}$. Comme on le voit, il ne s' agit que des «autorités théologiques» n'importe lesquelles, mais de fondateurs de la discipline (Paul et Augustin) et de principaux théologiens parisiens. En fait, le remerciement de Charlier s'adresse à Dieu, aux créateurs de la théologie chrétienne que représentent les docteurs nommés et, finalement, à une école particulière innommée, sans doute celle où Charlier tenait son discours, donc celle du collège de Cambrai ${ }^{12}$. Quant aux sources et auctoritates philosophiques, on les trouvera dans le texte même du Commentaire. Cependant, pour donner un bref aperçu, je prendrai en considération la quaestio collativa du Premier Pricipium de Charlier, en me limitant d'un côté à Aristote et quelques-uns de ses commentateurs (Eustrate, Albert le Grand, l' Aquinate), et de l' autre à Platon, Proclus, Liber de causis, le Ps.-Denys l'Aréopagite et Boèce, puis les trois philosophes arabes: Avicenne, Alfarabi (al-Farabi, Farabi) et Averroès, ainsi que du côté de la philosophie juive Raby Moys, c' est-à-dire Moïse Maïmonide ${ }^{13}$.

à la note 212, ajoute du poids à sa lecture de d' Ailly, le texte de celui-ci, dense des citations d'Aristote et du Commentateur, visiblement ne laisse aucune place à Proclus. L'idée du bien est alors connue de tous les philosophes et tous les théologiens. Omont 1919, p. 109120 (texte en ligne), ne note pas Proclus.

11 Jusqu'à présent, seul M. Zahnd 2016 (p. 101 et l'édition), s' est servi du cod. 205 de la Bibl. munic. d' Angers, inconnu de la littérature consacrée à Charlier.

12 Kaluza 2015, p. 332, 336-345 (texte); Zahnd 2016, p. 87-93, 101-105 (texte).

13 Dans la mesure de mes possibilités j' indique dans les notes l' origine des citations: ouvrage originale, florilège ou le médiateur. Je ne mentionne qu'à titre d'une rareté la seconde définition de Dieu provenant du Liber XXIV philosophorum: Deus est spera intelligibilis... 
Le calcul provisoire que j' ai fait en préparant la présente contribution, montre qu' une seule autorité philosophique domine la question collative, celle d'Aristote de la Métaphysique et de l'Éthique à Nicomaque, avec plusieurs autres ouvrages rappelés occasionnellement. Les trois philosophes arabes sont très rarement évoqués. La tradition platonicienne est, à son tour, dominée par Proclus, auteur de l'Elementatio theologica, suivi de près par l'Aréopagite avec le De divinis nominibus et, finalement, par Boèce, dont trois ouvrages sont pris en considération: De consolatione, De hebdomadibus et De Trinitate. Le Liber de causis semble un peu en retrait, mais moins que Platon.

Le Commentaire de Gilles Charlier est déjà décrit tant par le P. Victor Doucet que par Stefan Swieżawski ${ }^{14}$, il ne me reste alors que de rappeler quelques points principaux. Il est le fruit de l' enseignement conduit, comme cela a déjà été dit, en 1416-1417 et clos le 28 juin 1417. Le texte complet du Commentaire est conservé exclusivement dans ces deux manuscrits en parchemin, 958 et 959 de la Bibliothèque Mazarine à Paris, dont le premier, Mazar. 958, contient les Principia I et II avec les commentaires aux deux premiers livres des Sentences, et le deuxième, Mazar. 959, garde la suite: les Principia III et IV avec les commentaires sur les mêmes livres. M. U. Zahnd vient de révéler que le ms. Angers, Bibl. municipale 205, contient aussi une copie des livres III et IV du Commentaire de Charlier ${ }^{15}$.

La quaestio collativa, sujet principal de ce travail, constitue la deuxième partie d'un Principium des Sentences. Sa première partie, éminemment rhétorique, était obligatoirement un éloge du Maître Pierre Lombard et de quatre livres de ses Sententiae. Le caractère doctrinal de la question collative changeait d'une époque à l' autre, mais, presque toujours, cette pièce introductive soulevait des interrogations importantes de nature théologique, ontologique ou épistémologique. Il est aussi à remarquer que chaque collative réserve obligatoirement une place à la polémique avec quelques sententiaires concourants (socius, magister concurrens, reverendus etc.). Les noms des concurrents de Gilles Charlier sont connus: dans sa question du premier Principium il discute avec Gilles de Priches et Jean de Atrio, les deux de la nation picarde, à laquelle appartint aussi

(au lieu de infinita); por. Mazar. 958, question sur le Prologue Utrum theologia sit scientia homini necessaria ad salutem, f. 8 $8^{\text {rb }}$; Il Libro di ventiquattro filosofi, p. 56-57 et pour les sources Le Livre des XXIV Philosophes 1989, p. 93-96.

14 Doucet 1939, p. 405-442, part. p. 412-413; Swieżawski 1961. Les manuscrits Angers, Bibl. municipale, 204 et Troyes, Bibl. départementale 778, conservent le seul texte des commentaires aux livres I et II des Sentences. Cf. Doucet 1939, p. 405-442; Swieżawski 1961, p. $78-79$. 
Gilles Charlier ${ }^{16}$. Le premier Principium commence donc ainsi dans le Mazar. 598 , f. $1^{\text {ra }}$ :

Veteres mihi praebuere patres rectam, securam semitam, qua pergo: laudibus dignis Petrum celebrarunt doctum et praesulem Pariseorum. Et ego fidei pugiones et inclita theologorum luminaria universaliter famata per orbem 'post terga metentis ${ }^{17}$, si quo $\langle$ dam $\rangle$ modo valeam 'stipulas colligere $^{18}$ ' et 'micas de mensis dominorum cadentes ${ }^{19}$ ' avido $\langle$ or $\rangle \mathrm{e}$ sumere, disposui.

Inprimis itaque sacrum iaciam et imputribile fundamentum, scriptum $3^{\circ}$ Regum, $2^{\circ}$, alias per me sumptum ${ }^{20}$, ut ordinatius erat oratio: 'Currus Israel et auriga eius ${ }^{21}$.

«Char d'Israël et son attelage!».Émis par Élisée regardant le prophète Élie partir dans le char de feu, ce cri constitue ici le thème biblique des quatre livres des commentaires de Charlier. Et comme tel, il apparaît en tête du chacun des quatre Principia. Les prémices déroutent par le langage codé ou métaphorique, dont le sens se laisse comprendre ainsi. Rempli de sagesse, auriga noster ou le conducteur du char (currus), n' est autre que Pierre Lombard. Ce «char de sagesse» appartient au Maître du conducteur, Jésus Christ. C' est à Jésus que s'adresse alors la prière pour qu'il daigne accorder à Gilles et à ses auditeurs l'intelligence claire de cette sagesse qui remplit le char, c' est-à-dire des livres des Sentences. L'éloge du savant théologien et de son ouvrage, déjà annoncé, se termine au f. $2^{\text {ra }}$ :

Ad cuius vitae intelligentiam claram nos dignetur assumere suae sapientiae curru dominus aurigae nostri, Iesus Christus, verus filius Dei, qui est benedictus in saecula saeculorum. AMEN.

Suit immédiatement la première question collative:

Expeditus de primo venio ad secundum, ubi iuxta theuma collationis hunc formo titulum quaestionis: Utrum currus aurigae desiderium boni

\footnotetext{
16 Swieżawski 1961, p. 83 .

17 Jer. 9.22: Et cadet... quasi foenum post tergum metentis.

18 Ex. 5.7: sed ipsi vadant et colligant stipulas.

19 Matth. 15,27: nam et catelli edunt de micis quae cadunt de mensa dominorum suorum.

20 Est-ce une allusion à un commentaire biblique de Gilles Charlier? Un tel ouvrage aurait dû précéder celui de Sentences, mais on ignore son existence.

$21 \quad 2$ Reg 2.12 et 13.14. La traduction est de la Bible de Jérusalem.
} 
camerae civibus attulerit, id est: Utrum bonitas Dei omni creaturae desiderium sui indiderit ${ }^{22}$. Arguitur quod non. Desiderium fundatur in similitudine, Boethius, Regula viII libri De ebdomadibus: 'Omnis similitudo est appetenda... ${ }^{23}$.

La collative achevée au f. $7^{\text {va }}$, le Principium passe au $3^{\text {e }}$ point principal, pour s'achever immédiatement:

Quantum ad $3^{\mathrm{m}}$, de honore impenso collegio Cameracensi et huic scholae in persona mea modica, Deo et vobis humilime regratior ${ }^{24}$.

\section{2 \\ Dieu comme Bien suprême}

L'intitulé de la question pose ainsi le problème: la Bonté divine a-t-elle inspiré (a-t-elle donné) à tout être créé le désir d' elle-même? (Utrum bonitas Dei omni creature desiderium sui indiderit). La question considère donc Dieu comme la Bonté créatrice et inspiratrice, selon les deux aspects de son activité créatrice et inspiratrice. Autrement dit, celui qui crée, distribue dans les créatures un appétit, c' est-à-dire un désir de lui-même. On y voit déjà l' annonce d'un double mouvement, celui qui avance, lequel constitue l'être, et celui qui provoque son retournement par le désir. C'est ainsi qu' au point de départ Charlier suggère déjà l'importance de la double causalité divine: efficiente et finale ${ }^{25}$.

Suivent alors trois arguments en faveur de la réponse négative (quod non), puis les arguments en faveur de la thèse soutenue dans la question (ad oppositum) et, finalement, la division de la question. Sa structure est bipartite où la première partie traite de «la Bonté divine et de ce qu' elle a imprimé sur

22 Il me semble que le syntagme omnes creaturae traduit en langage plus claire le descriptif cives camerae.

23 Boethius, Quomodo substantiae, reg. IX (éd. Moreschini, p. 188, 44-45): Omnis diversitas discors, similitudo vero appetenda est.

24 Les quatre Principia de Charlier sont construits de façon identique. La formule de gratitude termine chaque Principium, cf. Kaluza 2015, note 18.

25 L'appétit causé par la Bonté divine permettra à Charlier de soutenir, contre Gilles de Priches, que l' amour nécessairement suivra la connaissance de Dieu. Cf. Mazar. 958, f. $5^{\text {ra }}$ : Sequitur collative quod divinam essentiam clare in patria visam voluntas non potest non diligere, ipsa enim divina essencia alliciat in infinitum voluntatem. Istud pono contra magistrum meum reverendum magistrum Egidium de Priches, qui oppositum posuit in quodam corollario sue 4 proposicionis. Dixit enim in forma quod possibile est creaturam racionalem Deum clare videre et ipsum non diligere. 
les facies des créatures: leurs formes et leurs aspects extérieures», alors que la partie suivante soulève la question du mal, lequel s'oppose au bien. Si cette deuxième partie est très courte, la première, en revanche, se développe en trois points, lesquels concernent (a) la Bonté divine envisagée en elle-même; (b) le premier effet de cette même Bonté créatrice qui est une capacité naturelle (aptitudo naturae) du créé de recevoir le bien dans son être, car, précisément, le bien même commence déjà par cette disposition réceptive; (c) le deuxième effet de la Bonté créatrice qui est une prédisposition et un penchant (tendentia) de la nature créée vers le bien ${ }^{26}$. Cela justifie la question portant sur la présence dans la créature universelle du désir du bien. Vient ensuite la seconde partie de la question qui soulève, comme on l'a dit, le problème du mal. L' architecture de la question est donc simple et transparente Voici son schéma:

I partie: de bonitate Dei et impressione eius super facies creaturarum;

(a) de bonitate Dei in se;

(b) de primo effectu eius qui est aptitudo naturae ad bonum in qua iam inchoatur ipsum bonum;

(c) de secundo effectu qui est inclinatio naturae et tendentia in bonum; II partie: de malo quod bono opponitur ${ }^{27}$.

\section{(a) Bonitas Dei in se}

La division de la question suppose une précise distinction des notions: Bonté en tant que telle et son «action», dont les principaux «effets» sont énumérés; son impression sur les créatures; l'aptitude de chaque nature créée à recevoir le bien; l'inchoation ou le commencement du Bien même a déjà lieu dans l'aptitude. Ce point concernant le premier effet de l'action créatrice de

26 Mazar. 958, f. $2^{\text {rb }}$ : His positis accedo ad positionem quaestionis in qua erunt duo puncta: primum erit de bonitate Dei et impressione eius super facies creaturarum, secundus erit de malo, quod bono opponitur, 'opposita enim iuxta se posita magis elucescunt,' $3^{\circ}$ Tho〈pico〉rum. In primo puncto tres sunt uno contextu videnda: primum est de bonitate Dei in se; secundum de primo effectu eius qui est aptitudo naturae ad bonum, in qua iam inchoatur ipsum bonum; tercium erit de secundo effectu qui est inclinatio naturae et tendentia eius in bonum. La référence aux Topiques est erronée, cf. Auctoritates Aristotelis, p. 245, n. 176; cfr. Aristoteles, Soph. el., 174b 5-6, p. 33, 17-19. Elle est aussi, et pour deux raisons, incorrecte, parce que la règle d' Aristote oppose deux propriétés (p. ex. deux couleurs, deux grandeurs etc.), alors que la Bonté divine ou le Bien en soi (considérés comme deux noms de Dieux) ne signifient aucune propriété et parce qu'il n' existe aucun terme contraire ou directement opposé à Dieu. La seconde partie de la question exclut une telle opposition et situe l'opposition entre les effets de la Bonté; cf. note suivante.

27 Mazar. 958, f. $6^{\text {rb }}$ : Pro $2^{\circ}$ puncto, ubi brevissime de malo agendum est, sit haec unica conclusio: Malum non est naura aliqua. 
la Bonté divine correspond aux effets de la première et nécessairement intérieure action du Bien comme tel. Les seconds résultats de l' action du Bien sont remplis de sagesse, marqués par l' inclinaison de la nature et par la tendance à recevoir un bien:les deux mots inclinatio et tendentia sont ici presque synonymiques.

Lorsque le texte parle de la nature et de ses caractères ou qualités, telles que aptitudo, inclinatio, tendentia, il parle de la nature créée. La signification du terme natura est alors très proche, sinon identique à celle de creatura. Ce qui nous attend donc c' est l' examen du Bien dans sa double manifestation: d'un côté le Bien en soi, actif en raison même de la Bonté qui le «constitue», et, de l'autre côté, les traces de cette activité divine laissées dans les créatures, c' est-à-dire les différents biens créés et participés. Alors, même si la question portant sur le mal est annoncée, le mal n'existant pas, elle a comme fonction de faire briller encore plus l'idée du Bien. Le Bien est codé dans la nature créée, comme tel il prend la forme de l'aptitude naturelle au bien, dont le penchant et la prédisposition ne sont que des manifestations inscrites dans l'être créé. À sa manière, l' ipsum bonum est donc présent dans la nature créée: il est là comme son fondement ontologique. On s' aperçoit alors pourquoi la catégorie principale de la pensée de Gilles Charlier dans sa question collative est le Bien, principe de toute action, connu dans les deux aspects mentionnés.

Le premier point, portant sur la Bonté divine comme telle, est fondé sur une seule conclusion à partir de laquelle Charlier déduira sept corollaires ou propositions. Voici d' abord la conclusion: «Dieu est par essence la bonté intégrale et parfaite ${ }^{28}$.

Sa première partie: que Dieu est la bonté par essence, est confirmée par une autorité scripturaire, celle de l'évangile selon Matthieu, 19.17: Nemo bonus, nisi solus Deus; et par une autre, philosophique qui est la proposition 121, légèrement écourtée, de l'Elementatio theologica de Proclus: «Tout dieu (ou tout divin) a la bonté pour essence»(Omne divinum essentiam habet bonitatem $)^{29}$. L'argument rationnel s' appuie sur la distinction entre le bien per se et le bien participé. Or, Dieu ne peut pas participer le bien, car il ne serait plus le premier bien. Il est ce bien qui est son essence ${ }^{30}$.

28 Mazar. 958, f. $2^{\text {rb }}$ : Pro primo puncto sit haec unica conclusio:Deus est bonitas per essentiam plena et perfecta.

29 Proclus, El. th., propos. 121: Omne divinum existentiam quidem habet bonitatem, (Boese. p. 6o); trad. Trouillard, p. 132: «Le divin tout entier détient à titre subsistant la bonté». 
Quant à la deuxième partie de la conclusion, affirmant que la bonté divine demeure intégrale et parfaite, elle est d'abord confirmée par trois autorités: une scripturaire (Ex. 33.19: «Je te montrerai toute ma bonté»), l' autre théologique (Denys l' Aréopagite, De divinis nominibus, chap. $5, \S 4$ : «Dieu n' est pas un existant de quelque manière, mais il a pris en soi tout être absolument et sans limite $\left.{ }^{31} \gg\right)$, et la dernière philosophique (Métaphysique d'Averroès): «À Dieu ne manque aucune perfection que l'on trouve dans un genre quelconque» (Nulla perfectio Deo deest que inueniatur in aliquo genere $)^{32}$. Succède ensuite et pour terminer un autre argument de raison, mais, en fait, emprunté à Aristote. Une perfection appartenant à la nature d'une chose (et ne dépendant d' aucune autre cause) ne peut être enlevée à cette chose et ne peut lui être ajoutée sans changement de sa nature et donc de sa perfection, car on ne peut pas amoindrir ou augmenter sa nature sans la changer. Les objets mathématiques l'illustrent parfaitement, car même une unité ajoutée ou enlevée à un chiffre donné, change la nature de ce chiffre, p. ex. $5+1$ et $5-1$ n'est plus 5 . Il peut arriver, cependant, par défaut d' une cause quelconque, que la nature demeurant intacte, on constate une perte de la perfection. Cela ne peut jamais avoir lieu en Dieu, car la bonté lui appartient de par sa nature et, comme l'affirme la première partie de la conclusion, la bonté divine reste à jamais intégrale et parfaite ${ }^{33}$.

Nous passons aux propositions.

〈1. $\rangle$ La première proposition. Il n'y a rien en Dieu qui ne soit la bonté (in Deo nihil est quin sit bonitas), autremant dit tout ce qui est en Dieu, est sa bonté et il n'y a rien d'autre. La démonstration du corollaire fait appel à la distinction boétienne entre quod est et esse, entre l'étant et l'être: «Ce qui est (quod

tive dicitur bonum. Sed Deus non est bonum per participacionem, sic enim non posset esse primum bonum. Igitur est bonum per essenciam.

31 Mazar. 958, f. $2^{\mathrm{rb}}$ : Deus non quodammodo est existens, sed simpliciter et incircumscriptive totum esse in se ipso accepit. Cf. Denys l' Aréopagite, De div. nom. v, 4; Dionysiaca, I, p. 333334 .

32 Cf. Les Auctoritates Aristotelis, p. 126, n. 138 qui renvoie à Averroès, In Metaph., v, com. 21, f. 131 в.

33 Mazar. 958, f. $2^{\text {va }}$ : Racione sic. Quod alicui convenit ex sua natura et non ex alia causa, minorandum in eo et deficiens esse non potest, quia a natura rei nihil subtrahi potest aut addi, nisi natura varietur. Sunt enim species rerum, sicut numeri, $8^{\circ}$ Metaphysicae, in quibus unitas addita aut remota variat speciem; et si natura integra manente, aliqua perfectio inveniatur deficienter, manifestum est quod est ex defectu alicuius alterius causae. Sed bonitas divina convenit ei per suam naturam, ut dixit prima pars conclusionis, ergo ipsa non est diminuta, sed plena et perfecta. Pour Aristote, cf. Meta., VIII 3, 1043b36-1044a2, A.L., XxV.2, p. 162, 19-22. 
est) peut avoir quelque chose en dehors de ce qu'il est lui même. Au contraire, l'être même (esse) n' a rien d' autre qui se mêle à lui en dehors de lui-même ${ }^{34}$ ». En remplaçant le quod est par le substantif bonum et l'esse par bonté, Charlier transforme de la sorte la règle boécienne: «De la même manière la bonté ne peut avoir rien d'extérieur, quoique le bien même puisse avoir quelque chose d'extérieur» et trouve un rapport logique entre cette reformulation et la fameuse définition avicennienne de l'équinité: equinitas est equinitas tantum: «C' est pourquoi Avicenne dit: 'L'équinité est seulement équinité et rien d' autre ${ }^{35}$ ' . Et une nouvelle explication inspirée par le De trinitate de Boèce: «Une forme simple ne peut être le sujet [de quelque chose], mais Dieu, nous le savons déjà, est sa bonté [et rien d' autre]. Alors en Dieu il n'y a que la bonté » ${ }^{36}$. Alors Dieu doit être compris comme l' être pur (esse), définit comme bonté pure (bonitas), comme une «essence sans plus», une forme simple. Il sera juste de voir dans ce premier corollaire l' extension de la spéculation sur le caractère de l'essence divine qu' est la bonté où il serait impossible de distinguer quoi que ce soit.

Cela une fois établi, Gilles Charlier montre dans quel sens la doctrine qu'il enseigne est celle de Université de Paris. La question collative étant un acte scolaire, Charlier, au nom de cette pureté de la notion de Bonté divine attaque quelques théologiens innomés qui osent, en matière de divinis, appliquer la distinction formelle dite ex natura rei et affirmer que la sagesse divine n' est pas la bonté divine: Ex quo patet falsitas dicentium: ex natura rei in divinis sapientiam

34 Boèce, Quomodo substantiae, Reg. Iv: Id quod est habere aliquid praeterquam quod ipsum est potest; ipsum vero esse nihil aliud praeter se habet admixtum (éd. Moreschini, p. 188). La traduction est d' Alain de Libera 1993, p. 251; voir aussi p. 250-252.

35 Avicenne, Liber de philosophia prima sive scientia divina, II, tr. V, c. 1, p. 228, 31-36: Equinitas etenim habet definitionem quae non eget universalitate, sed est cui accidit universalitas. Unde ipsa equinitas non est aliquid nisi equinitas tantum; ipsa enim in se nec est multa nec unum, nec est existens in his sensibilibus nec in anima, nec est aliquid horum potentia vel effectu, ita ut hoc contineatur intra essentiam equinitatis. On parle alors de «l'indifférence de l' essence».Cf. Libera 1999, p. 515 sq. P. Vignaux présente l' usage de cette conception avicennienne dans une étude sur «L'Être comme perfection chez François de Meyronnes». Cf. Vignaux 1976, p. 265sq.

36 Mazar. 958, f. $2^{\text {va }}$ : Sequitur primo quod in Deo nihil est quin sit bonitas. Probatio, et si ipsum quod est aliquid habere possit extraneum, non tamen ipsum esse, Boethius, De hebdomadibus, regula 3 (!), ita bonitas nihil extraneum habere potest, quamvis tamen ipsum bonum extraneum aliquid habere possit. Ideo Avicenna: 'Equinitas est tantum equinitas', et Boethius libro De trinitate: Forma simplex subiectum esse non potest, sed Deus est sua bonitas, ut dixit prima pars conclusionis, ergo in eo nihil est nisi bonitas. Boèce, De sancta trinitate, éd. Moreschini, p. 170, 104-106: Neque enim subiectum fieri potest: forma enim est, formae vero subiectae esse non possunt. 
non esse bonitatem etc. Il s' agit à l'évidence des scotistes - formalizantes, critiqués déjà par Pierre d'Ailly et surtout par le chancelier Gerson les prenant pour des platoniciens de son temps ${ }^{37}$.

〈2. $\rangle$ La seconde proposition: la bonté divine est infinie, car elle n'est pas reçue par la matière, laquelle la limiterait nécessairement. Elle n'est donc pas une forme par participation, mais par essence. Et alors elle n'a pas de bornes. C'est pourquoi toute forme reçue est une forme par participation, «mais la bonté divine n'est pas bonne par participation, mais essentiellement $^{38}{ }^{»}$. Charlier évoque ici un texte de Denys, que je n'ai pas réussi à identifier.

〈3. La troisième proposition affirme que la Bonté divine n' est qu'une: tantum est una. L'être d'une forme infinie qu' est cette Bonté possède toute la perfection de sa nature (extra ipsam nihil est de illa natura). Et si elle possède toutes les perfections, alors toutes les perfections de cette nature se trouvent dans une seule bonté (tota bonitas infinita et subsistens est in una, igitur non sunt plures bonitates infinitae). La suite du corollaire s' appuie sur l'autorité d'Aristote et sur celle de l'Elementatio theologica de Proclus deux fois citée: d'abord pour rappeler que toute la pluralité des participants ont la même unité à l'origine (propos. 5), puis pour affirmer que les êtres provenant d' une unité, «procèdent de leur monade propre» (propos. 110, comm. $)^{39}$. Le propos attribué à Aristote

37 Mazar., 958, f. $2^{\text {va }}$ : Ex quo patet falsitas dicentium: ex natura rei in divinis sapientiam non esse bonitatem, et sic de aliis. Quod enim dicitur de aliquo 'non inquantum huiusmodi' convenit ei per accidens racione subiecti et non racione formae cui attribuitur... Cf. Kaluza 1988, s. 127 sqq.

38 Mazar. $95^{8}$, f. $2^{\text {va }}$ : Sequitur $2^{\circ}$ quod bonitas Dei est infinita. Probatio. Forma finitur per materiam recipientem, ideo omnis forma recepta est participative talis. Neque sensibilia adaequare et comprehendere possunt intelligibilia, neque potentia actum, secundum Dionysium. Sed Dei bonitas non est per participationem, sed per essentiam. Ergo est infinita.

Mazar. 958, f. $2^{\mathrm{va}}$ : $\langle 3$.$\rangle Sequitur ultra quod tantum est una. Probatio. Forma infinita subsis-$ tens tota habet perfectionem illius naturae, ergo extra ipsam nihil est de illa natura; ergo tota bonitas infinita et subsistens est in una, igitur non sunt plures bonitates infinitae. Ideo Aristoteles $5^{\circ}$ Metaphysicae: 'substantia cuiuslibet rei semel est,' et Proclus propositione 5: 'Omnis multitudo secunda est ab uno' (Boese, p. 4) et 111: 'Ab uno et ad unum procedit quod est ex propria monade' (Boese, prop. 110, comm. p. 56,12-13). Et nisi esset prima bonitas una, unumquodque esset plura bona et nihil unum aut simpliciter aut analogice. Cette dernière conclusion tirée du $3^{\mathrm{e}}$ corollaire prouve bien que Charlier s' inspire aussi du commentaire de la proposition 5 de l'Elementatio. Aristote analysant la catégorie quale introduit la notion de différence secundum substantiam, laquelle différencie, entre autres, les immuables objets mathématiques, comme p. ex. les nombres cardinaux. Ceux-ci, en dehors de leur quantité possèdent une qualité, celle qui les constitue une seule fois dans leur substance. Aristote, Meta., v 14, 1020b7-8, AL xxv.2, p. 25-27: nam substantia cuiuslibet quod semel, ut sex non bis tres sunt sed semel; sex enim semel sex. 
(substantia cuiuslibet reisemel est) déforme la pensée du Philosophe qui parlant des objets immuables mathématiques appelle 'substances' les nombres cardinaux, constitués une fois pour toute dans leur qualité, laquelle ne résulte donc d' aucune opération mathématique. Aristote latin dit ceci: Substantia cuiuslibet numeri semel est.

〈4. La quatrième proposition affirme que Dieu est le bien suprême (summum bonum). Cela découle du corollaire où il est dit que la bonté divine est infinie. Puis, ce qui est affirmée selon l'essence est plus vrai que ce qui est dit par participation. Or Dieu est verissime bonum, et donc summum bonum, parce qu'il est bon par son essence. Dans le premier livre du De trinitate, saint Augustin affirme que «la Trinité des Personnes divines est le bien suprême qui

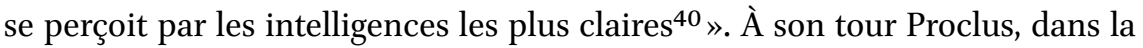
proposition 8: «Le bien primordial, qui n' est rien d' autre que le bien, devance (praecedit) tous les êtres qui, de quelque manière que ce soit, participent au bien $^{41}$ ». Ma traduction est pourtant incertaine, car je ne sait pas comment Gilles Charlier a-t-il compris le verbe praecedo, lequel, en cet endroit précis correspond au verbe grec hegéomai rendu, dans la traduction greco-française de Jean Trouillard, par dominer. Un deuxième embarras est causé par une petite omission de deux mots dans le propos de Proclus cité par Charlier - cette omission peut être voulue. Je la signale dans la note.

〈5. La cinquième proposition exclut tout mal de Dieu, d'abord parce que son bien est infini, donc sans limites, et puis parce que ce qui est en Dieu, il y est essentiellement ${ }^{42}$.

40 Je n'ai trouvé que le propos sur l' expression purgatio mentis nostrae, cf Augustin, De Trinitate, I, i, 3, p. 90-92.

41 Mazar. 958, f. $2^{\mathrm{va}}$ : Sequitur ultra quod Deus est $\left(2^{\mathrm{vb}}\right)$ summum bonum. Patet ex infinitate suae bonitatis etiam quod per essentiam diciturverius quam quod participative. Sed solus Deus est per suam essentiam bonus, ergo est verissime bonum; ideo summum. Unde Augustinus I De trinitate: 'Trinitas divinarum Personarum est summum bonum, quod purgatissimis mentibus cernitur', et Proclus propositione 8: 'Omnia qualitercumque participantia bono praecedit quod prime bonum 〈et quod〉 nihil est aliud quam bonum? Sequitur quod Deus est bonum omnis boni. Patet ex dictis. Proclus, Elementatio theologica, prop. 8, éd. Boese, p. 7. Voir L'index latino-graecus de l'édition citée, p. 125, s.v. precedo et la traduction de Trouillard, p. $65-66$.

42 Mazar. 958, f. $2^{\mathrm{vb}}:$ Sequitur ultra quod in Deo nullum potest esse malum. Patet, quia est bonitas infinita et unumquodque infra suos terminos comprehendit, ut nihil extraneum capere possit. Ideo Prima canonica Jo. 20 (I Ioh 1,5) dicitur: 'Deus lux est et in eo tenebrae non sunt ullae. Et praeterea quidquid in Deo est, est essentialiter (ms. eternaliter). Si ergo malum esset in Deo, esset malum per essentiam. Sed hoc est impossibile, ut infra patebit. 
〈6. La sixième proposition: rien n' est contraire à la bonté divine. Les formes contraires ayant en commun la nature et ainsi un genre commun, appartiennent au même genre. Mais Dieu ne se limite à aucune forme générique et par conséquent rien n' est lui contraire ${ }^{43}$. C' est pourquoi Avicenne et Algazel affirment, dans leurs Métaphysiques, que rien ne peut être égal au Premier, lequel n'a aucun consort, aucun être similaire qui lui soit opposé ou même adverse ${ }^{44}$. Cela une fois établi, Gilles Charlier indique la fausseté tant de la doctrine de Mani et de ses prédécesseurs jusqu'à Empédocle, que de la liste des principes opposés, composée par des pythagoriciens et transmise par Aristote ${ }^{45}$. Telles sont les conséquences théologiques et philosophiques qui découlent de cette sixième proposition.

〈7. La septième proposition soutient qu'en Dieu il n'existe aucune puissance passive (potentia passiva), car la puissance séparée de l'acte est la racine du mal. L'idée suit une maxime d'Avicenne, connue et répétée quelquefois par Albert le Grand, selon laquelle bonum est indivisio actus a potentia. En revanche, en admettant en Dieu une puissance passive, nous accepterions en Lui le mal, en nous mettant encore une fois en contradiction avec les philosophes Arabes, Avicenne et Algazel, pour qui, en delà de la Lune le mal n' existe pas $^{46}$. Il s' agit d'un peu plus que ne le dit Charlier. En effet, d'après Albert le

43 Mazar. $95^{8}$, f. $2^{\mathrm{vb}}:$ Sequitur ultra quod bonitati divinae nihil est contrarium. Probatio. Contrariae formae sub unius generis ambitu continentur, quia et illarum una est communis natura, et ita unum commune genus. Sed Deus nulla generis figura clauditur, ergo nihil est ei contrarium.

44 Mazar. 958, f. $2^{\mathrm{vb}}$ : Ideo Avicenna et Algazel in suis Metaphysicis dicunt primum non habet consortem, comparem, similem, oppositum aut adversum. Avicenne, Liber de philosophia prima, X, c. 2, t. II, p. 534, 29-30: Non oportet autem ut detineat eos circa cognitionem alicuius rei de Deo, nisi circa hanc scilicet quod est unus, vivens, non habens similem; et p. 535, 47-50: Immo oportet ut insinuet eis gloriam Dei et magnitudinem eius [...] et cum hoc dicat eis quod non est sibi aliquid compar vel simile.

45 Mazar. 958, f. $2^{\mathrm{vb}}$ : Patet ibi falsitas Manicheorum (a Manic haeretico de regione Persidis sic dictorum), ponentium duo principia prima, unum Lucis, alterum Tenebrarum. Et iste Manic a Marcho haeretico et inde a Marchianistis sumpsit hanc haeresim. Et isti adhuc prius originem sumpsisse videntur a pictura speciali Empedoclis, qui posuit duo principia pincipalia, litem et amicitiam. Similiter patet falsitas picturarum ponentium duas coelementationes primas, unam bonorum aliam malorum, ad invicem oppositas, ut recitat Aristoteles $1^{\circ}$ Metaphysicae. Respectivement Aristote, Meta., I 4, 984b33-985a1o, AL XXV.2, p. 15, 23-16, 11 ; et Meta., I 5, 986a22 sqq., AL XXv.2, p. 18, 22-19, 4.

46 Mazar., $95^{8}$, f. $2^{\mathrm{vb}}$ : Sequitur ultra quod in Deo nulla est potentia passiva - est enim potentia distans ab actu radix mali. Ideo Avicenna:Bonum est indivisio actus a potentia. Si ergo in Deo esset potentia (quae non esset actus), qualis est potentia passiva, in Deo posset esse malum, cuius oppositum patuit, et contra Avicennam et Algazelem dicentes: ultra orbem Lune non est malum. Respectivement Avicenne, Liber de philosophia prima, IV, chap. 2, t. I, p. 212, 
Grand, l'étant par puissance est la racine de la puissance passive, et l'étant par acte racine de la puissance active. Alors, si en Dieu il n'y a pas de puissance passive, c' est parce que Dieu n' est pas un étant en puissance. L'étant in potentia n' est qu'une modalité de l'être (modus entis), seul l'ens est l'étant au sens absolu ${ }^{47}$. Par ailleurs, dans son De causis et processu universitatis, Albert affirme: «De lors que, dans le v livre de la Métaphysique, on définit la puissance de deux manières, il est reconnu que la puissance passive ne peut d'aucune manière exister dans le premier ${ }^{48}$.

<8. La dernière proposition de cette première partie de la question énumère les «qualités du Bien suprême», qui est très riche et très beau (les deux vont ensemble), le plus parfait, donc existant per se, très joyeux et possédant une vie heureuse. Les confirmations de ces attributs étant faites rapidement, elles constituent un bouquet de fleurs (flores ou auctoritates), parmi lesquels il faut signaler l' apparition d' une nouvelle auctoritas de Charlier, à savoir le Commentaire de Michel d'Ephèse sur le livres IX et $\mathrm{x}$ de l'Éthique à Nicomaque, traduit par Robert, évêque de Lincoln. C' est à propos de la délectation, sujet principal du livre $\mathrm{x}$, que Michel d'Ephèse examine le rôle de la délectation dans notre agir: est-elle toujours le complément de l' action humaine? Michel répond en prenant l' exemple de la beauté (pulchritudo et decor) d'une jeune personne, dont les membres et les autres parties du corps composent un ensemble harmonieux, une commensuratio qui accompagne (coest) celle-ci au refleurissement de la nature: flos reflorescens et que Charlier exprime par refloritio naturae.

Sequitur ultra quod summum bonum est ditissimum per se, sufficiens, nobilissimum, pulcherrimum, laetissimum et vitae fecis. Quod ditissimum, in Psalmo $\langle 15,2\rangle$ : 'Bonorum meorum non eget' et alibi $\langle$ Ps. 49 12): 'Meus est orbis terrae'. Quod pulcherrimum: quia optimum; modo 'bonum laudatur ut pulchrum', Dionysius, 4 capitulo De divinis nominibus $^{49}$. Quod propinquitas nominis ostendit, in graeco bonum enim dici-

43-44: Potentia enim est imperfectio et effectus est perfectio, et bonum in omni re non est nisi ipsam esse in effectu; Ibid., IX, cap. 6, t. II, p. 498, 6-7: Omnes autem causae mali non inveniuntur nisi in his quae sunt sub orbe circulo lunae. Ainsi, la discussion engage la cosmologie avicennienne.

Albert le Grand, Metaphysica, t. I, v, tr. 3, c. 12, p. 25o, 32-5o. Les remarques d' Albert éclaire le texte d'Aristote (Meta., V 12, AL Xxv.2, p. 99-101), où le Philosophe donne les bases conceptuelles pour expliquer le changement (mutatio).

48 Albert le Grand, De causis et processu universitatis a prima causa, p. 37, 77-84.

49 Denys l'Aréopagite, De divinis nominibus, IV, 7 ; Dionysiaca, I, 178: Hoc bonum laudatur a sanctis theologis et sicut pulchritudo et sicut pulchrum (...). 
tur 'calos' per unum $l$ et pulchrum 'callos' per duplicem $l^{50}$. Quod laetissimum patet per Aristotelem $10^{\circ}$ Ethicorum: 'Perficit operationem propria delectatio'. Et Michael Ephesius: delectatio est refloritio naturae in naturali habitus operatione non impedita ${ }^{51}$. Sed Deus habet intellectualem operationem perfectam et non impeditam. Quod autem eius vita sit beata patet, quia perfectissima et laetissima ${ }^{52}$.

Toutefois, en nous rappelant les richesses du Bien suprême, ce dernier corollaire nous permet de passer au point suivant qui est, précisément, leur communication aux créatures et les effets produits par la largesse infinie, dont le premier et principal est l'aptitudo naturae ad bonum in qua iam inchoatur ipsum bonum.

\section{(b) Effectus Bonitatis}

Le second point de la question est construit selon le même modèle que le premier: on y lit une série des propositions, qui commencent par un sequitur quod et semblent découler soit l'une de l'autre, soit toutes d'un premier, inscrit en tête comme axiome ouvrant cette nouvelle suite des conséquences. Voici donc cet «axiome» et la raison qui le confirme d'une manière laconique: Suae bonitatis divitias Deus aliis a se communicat. Probatur, quod infinite bonus. Cette raison est immédiatement renforcée par l' autorité du Pseudo-Aréopagite affirmant dans le De divinis nominibus que «L' Amour divin ne laisse pas Dieu demeurer sans progéniture»(Divinus amor non sinit ipsum esse sine germine $)^{53}$.

50 Cf. Dionysiaca, I, p. 180: (...) unde et calos dicitur et sicut tota in totis congregans. Albert le Grand, Super Dionysii De divinis nominibus, IV, n. 77, p. 186, 20-32: Deinde ponit tertiam conditionem 〈pulchritudinis $\rangle$ (...). Cet important paragraphe s' achève par une critique de la traduction érigénienne: Unde et pulchrum apud Graecos dicitur 'kallos', quasi 'vocans', et nota, quod in hoc fuit deceptus alius translator: 'kallos' enim per duplex 'l' scriptum significat 'pulchrum' apud Graecos, per unum vero 'l' significat 'bonum, et ideo semper transtulit 'bonum' pro 'pulchro', et ideo in parte ista alia translatio est tota vitiosa.

51 Aristote, Eth. Nic., X 4, 74b31-75a2, AL, XXvi 1-3, p. 352, 22 et p. 353, 9-10: Perficit autem operacionem, delectacio, non habitus qui inest (...). Le texte est repris dans le Commentaire de Michel d'Éphèse, The Greek Commentaries, p. 363, 62-68; commentaire de Michel d'Éphèse, ibid. p. 372-373. Je me réfère à la p. 373, 35-39: Quemadmodum enim pulchritudo et decor, usque quo utique partium et membrorum ad invicem et ad totum salvatur commensuratio, coest ipsi - huic enim commensurationi supervenit et velut lux quaedam vel flos refulgens vel reflorens - sic delectatio semper coest operationi, potentissimae videlicet.

$5^{2}$ Mazar. 958, f. $3^{\text {vb-ra }}$.

53 Denys l' Aréopagite, De div. nom., IV, 8; Dionysiaca, I, 201: Et est divinus amor bonus boni propter bonum; ipse enim bonum operans existentium amor, in bono secundum excessum 
Gilles Charlier établit ainsi un lien discret entre la thèse néoplatonicienne sur le Bien suprême communicant le bonum à toutes les créatures et l'idée de la fécondité divine enracinée dans l' Écriture. Heimeric de Campo, qui, comme je le pense, fut présent lors de la discussion autour de la première quaestio collativa de Charlier, est revenu plusieurs fois sur ce thème de fécondité divine, en s'appuyant toutefois sur le verset d' Isaïa 66.9: Si ego qui aliis generationem do, ipse sterilis ero ${ }^{254}$ Albert le Grand, dont le commentaire suit attentivement la lettre du De divinis nominibus, trouve dans le passage IV 8, cité à l' instant, le langage imagé et métaphorique. Il l'admet pourtant en se référant discrètement au verset Gen 1,26 et en considérant les rationes similitudinis comme flores divini ${ }^{55}$.

Commencent alors deux longues séries d'explications et de précisions qui éclairent les résultats de la communication - Charlier dit "l' ensemencement" du bien par le Bien suprême. Prise dans un schéma général, cette communication est double, ainsi que son résultat. Le premier en est la coaptatio potentiae (ou naturae), c' est-à-dire l'établissement d'un rapport proportionné entre la puissance de la nature et de l' acte reçu. Il s' agit d'une sorte de proportion rappelant la commensuratio. Plus loin la coaptatio sera appelée aptitudo naturae vel potentiae ad bonum. L' autre résultat est fondateur dans le créé d' une inclinaison de cette-même puissance vers le bien ${ }^{56}$. Finalement deux passions les plus élémentaires et les plus générales correspondent aux deux résultats: l' amour au premier, le désir au second.

Charlier décide alors d'aborder le problème de premiers effets de la communication du bien à l'égard de chaque créature. Il la décrit comme action

praeexistens, non dimisit ipsum sine germine in se ipso manere. Cfr Albert le Grand, Super Dionysium De divinis nominibus, p. 214, 32-43.

54 Le verset Is 66, 9 est cité dans le Commentaire du Pater noster, cf. Heymeric de Campo, Testamentum doctrinale, pars II: Oratio Dominica, ms. Bruxelles, Bibl. Royale, ms. 1157175 (Catalogue de Van den Gheyn $\mathrm{n}^{\circ} 2177$ ), f. $14 \mathrm{1}^{\mathrm{va}}$ et pour la citation f. $14 \mathrm{1}^{\mathrm{vb}}$; cf. Kaluza 2011, p. 532-534. Heyemeric de Campo, Tractatus de naturali veritatis catholice analesy, p. 190.

55 Albert le Grand, Super Dionysium De div. nom., op. cit., IV, n. 120, p. 215,11-16: Ad primum vero dicendum, quod quamvis creatura procedat in diversitate essentiae a primo, procedit tamen sub rationibus similitudinis essentiae primae secundum imaginationem et vestigium; unde illae raciones sunt quasi quidam flores divini, et propter hoc potest congrue dici 'germen' creatura sui creatoris. Cf. Gen. I,26: Et ait $\langle$ Deus $\rangle$ : Faciamus hominem ad imaginem et similitudinem nostram. Cf. Libera 2005, p. 185-186.

56 Mazar. 958, f. $3^{\text {ra }}$ : Sed quia boni respersio in creaturis est duplex, secundum duplicem eius effectum - quorum unus est coaptatio et proportio ad suum bonum, alter inclinatio potentiae ad illud penes quod duae primae et communissimae passiones inveniuntur in omnibus modo suo - amor iuxta primum, desiderium iuxta secundum. Ideo primo de primo effectu (...). 
généreuse qui fixe dans les êtres leur caractère ontologique et leur accorde l' inchoatio boni, dont je n' ai trouvé aucune explication C' est ainsi qu' une forme exacte est plantée dans chaque chose ${ }^{57}$.

Ideo primo de primo effectu Primi boni in creaturis, scilicet aptitudine naturae vel potentiae ad bonum, infero quod super omne quod est Bonitas divina liberaliter se diffundit praefigendo rebus modum et mensuram, et in hoc boni inchoationem omnibus, quae sunt. Et sic in forma bona plantatur omne quod est, ut loquitur Dionysius.

Au moment même de la réception des biens mentionnés, la chose créée, richement dotée, commence de se tourner vers sa fin (in suum finem). Au début, quand son intelligence de l' inclinaison reste encore imparfaite, ce mouvement est faible: la créature ne sait pas encore que l'inclinatio doit correspondre à toute aptitudo naturae ou potentiae, dans laquelle l' inclinaison est fondée. Elle est là, comme une forme par laquelle on se dirige vers la fin ${ }^{58}$.

L'importance de la cause finale est immédiatement soulignée. En effet, la communication du bien ne dépend d'aucune manière de la cause efficiente, qui marque le moment initial des choses, mais directement de la fin, qui est le terme des choses. En revanche, c'est le bien dit boatus qui boat, autrement dit vocat, et attire les choses comme leur fin. Dieu retourne (convertit) vers lui, comme vers la fin toutes les choses par un tel appel. Un argument soutient cette opinion. Or, si tout agent agit en fonction d' une fin, la mise en ordre des choses par rapport à une fin est aussi large que l'exitus de choses opéré par l'efficiente autrement, on serait obligé d'admettre que l'exitus provient d'un principe fortuit, quelque part mal formé par la tension de la fin (intentio finis $)^{59}$.

57 Mazar. 958, f. $3^{\text {ra }}$. Les termes modus et mensura viennent de la théologie augustinenne, alors que inchoatio formarum dépend de la largesse créatrice. À propos du concept d' inchoatio, cf. Libera 2005, p. 165-166 et 178. L'allusion finale renvoie à Denys, De div. nom. IV, 4 ou V, 8; Dionysiaca, I, 166-167 ou 357-36o. Cf aussi Albert le Grand, Super Dionysium De div. nom. (cf. note 5 ), p. 162 , lemme du texte et comm. $57^{-58}$; p. $3^{23}$, lemme du texte et comm. 34 .

$5^{8}$ Mazar. 958, f. $3^{\text {ra }}$ : Sequitur ultra quod in illa receptione iam incipit res in suum finem converti, sed imperfecte et quasi materialiter, quia nondum intelligitur habere inclinationem in illum secundum totam aptitudinem in natura, ut tendat in finem, in qua fundatur inclinatio, quae se habet sicut forma qua tenditur in finem. Pour la notion de conversio, cf. Hadot 1999 .

59 Mazar. 958, f. $3^{\text {ra }}$ : Sequitur ultra quod diffusio boni pertinet ad finem et non ad genus cause efficientis. Patet ex dictis, efficiens enim inquantum tale non est rei terminus sed initium. Bonum vero boat, id est vocat et trahit omnia ad se tamquam ad finem. Ex quo patet quod 
S'agissant de la finalité et des causes finales, Charlier constate une multitude et une variation des fins, mais aussi le fait que ces fins sont hiérarchiquement ordonnés, avec au sommet, une fin médiane. L'existence de la fin ultime médiane au sommet des structures ou séries ordonnées des étants assure le transfert des richesses venant du Premier vers les étants. Car, en fait, tel est le chemin de toute la communication des richesses obtenues par participation. La communication des richesses par la fin médiane dans les séries ordonnées ne se fait pas à partir d'un trésor propre, lequel dans chaque cas est «emprunté», car obtenu par participation à la cause ultime. Empruntant à son tour un exemple à la médecine, Gilles Charlier illustre l'organisation d'une série des causes et effets. Alors, chaque diffusion du bien par une cause médiane et toute participation entre un effet et sa cause se font en vertu de la bonté divine qui est la fin ultime de tout ${ }^{60}$.

Le passage qui suit ne mérite pas ma mauvaise traduction ni paraphrase qui l'aurait privé de son caractère inattendu chez un théologien. Sans l'évaluer, je le donne tel quel ${ }^{61}$ :

Sed quia emanatio bonorum non solum pensatur secundum virtutem boni, eo quod sic in infinitum flueret inferius, sed etiam secundum ordinem rationis, cuius est praefigere formam et modum rebus, et sapientiae, cuius est ordinare, $1^{\circ}$ Metaphysicae, ideo processio bonorum et pulchrorum a Deo fit in creaturas secundum ordinem quaedam illustrando clarius, quaedam obscurius, $\langle$ ut $\rangle$ habet Aristoteles $1^{\circ}$ Celi ${ }^{62}$. Quem ordinem quidam theologi poetae subobscure tetigerant, dicentes quibusdam communicatam esse bonitatem in sempiternum, quia gustaverant nectar et manna, aliis tamen cum defectu, quia cibum istum et ambrosiam non

hec vocatio fit per hoc quod omnia Deus convertit in ipsum - manifestum est ex dictis. Et hoc arguitur sic. Omne agens agit propter finem, ergo ordinatio rei in finem ad tantum se extendit ad quantum exitus rei ab efficiente principio. Alioquin esset exitus rerum a principio casuali, quia in aliqua sui parte non esset informatus intentione finis. Cf. Libera 2005, p. $184-189$.

6o Mazar, 958, f. $3^{\text {ra-rb }}$ : Sequitur ultra, si aliquid citra Primum sit aliorum finis, sub fine $\langle$ ultimo $\rangle$, suas participatas divitias communicabit his, quae sunt ad ipsum non ex propriis thesauriis, sed ab ultimo fine mutuatis. Probatio, in omnibus finibus ordinatis finis ultimus est finis omnium praecedentium, ut si potio | conficiatur ut detur, datur ergo ut purgetur, purgatur ut extenuetur, extenuatur ut sanetur, sanitas est finis extenuationis, purgationis et confectionis. Sed Deus est ultimus finis omnium, nihil ergo nisi virtute bonitatis divinae influit divitias pertitae bonitatis. Ideo solius Dei actio est larga, liberalis et magnifica, ut habet Avicenna.

61 Mazar. 958, f. $3^{\text {rb }}$.

62 Auctoritates Aristotelis, p. 161, n. 30; Aristote, De caelo, I 9, 279a28-3o. 
gustaverunt, ut recitat Aristoteles $3^{\circ}$ Metaphysicae ${ }^{63}$. Alios poetas recitat Johannes Bocatii sic dixisse: Summus deus qui vocatur Demongorgon, $\langle$ qui $\rangle$ primo genuit Litigium in quo significatur rerum distinctio, consequenter Pan fratrem Litigii, ubi significantur naturales instinctus ad proprias operationes, consequenter tres filias, quas philosophi 'Fata', poetae 'Parcas' dicunt: Clotho, Lachesim et Atropos ${ }^{64}$.

Pour terminer ma présentation de la première Quaestio collativa de Gilles Charlier, présentation nécessairement partielle et trop superficielle, j' aimerais encore jeter un rapide coup d'oeil sur ses dernières conclusions, dont l'argumentation engage quelquefois l' autorité de Proclus.

La thèse centrale est celle-ci: lors du retour de la chose vers la fin, l' influence du Bien ne compose pas (= ne se met pas à composer une union avec) la chose, mais la simplifie. Or toute forme à la fin de son mouvement est plus simple qu' au milieu de la route et quand la chose est déjà acquise et unie à sa fin, la similitude de l'être premier et simple lui sera son ultime perfection ${ }^{65}$.

1. Parlant de l'écoulement du bien lors du retour d'une choses vers la fin, Charlier affirme: cet écoulement préserve les choses (salvat) dans leur être sans ses mêler avec elles (ista influentia res salvat in esse praeterquam eis commisceatur). L'argument en faveur de la première partie est emprunté à l'Elemantatio teologica, proposition 11, dit Charlier; en fait il s' agit du commentaire de la proposition $13^{66}$. La seconde partie de la conclusion est confirmée par la thèse connue de tous, selon laquelle «le flux divin ne communique en rien avec les créatures, car l'être de Dieu et son opération sont sa substance», et parce que Dieu est séparé des choses selon son quod est, il l' est aussi selon ses opérations ${ }^{67}$. Dans le cas contraire, l'influence de Dieu ne simplifie pas les choses. Charlier ajoute

63 Aristote, Meta., III 4, 100oa9-19, AL 25 2, p. 52, 20-53, 3. Cf. Albert le Grand, Metaphysica, p. 126-127. Cf. Thomas d'Aquin, In Metaph., III, lectio 11, n. 468.

64 Le texte de Boccace se lit en ligne: http://agoraclass.fltr.ucl.ac.be/concordances/boccaccio _genealogiae_ol/texte.htm. Introduction et premier chapitre.

65 Mazar., 958, f. $3^{\text {va }}$ : Sequitur ultra quod influentia boni quae est in retractu rei ad finem simplificat et non componit res. Patet ex dictis.

66 Mazar., 958, f. $3^{\text {va }}$ : Sequitur ultra quod ista influentia res salvat in esse praeterquam eis commisceatur. Probatur prima pars, unitate omnia salvantur et dispersio unumquodque facit exire a substantia, habet Proclus, propositione 11. Cf. Proclus, Elementatio theologica, éd. Boese, p. 10, prop. 13, 5-6: uno salvantur omnia (...).

67 Charlier revient sur le problème dans son Commentaire, livre I, dist. 37 (ms. 958, f. $94^{\text {rb }}$ ), question: Utrum Deus sic sit in rebus omnibus quod commisceatur eis aut non et quid sit ipsum commisceri rebus. Voir aussi note suivante et Swieżawski 1961, p. 81. 
ici l' autorité de Proclus, la proposition 122: «Tous ce qui est divin (Omne divinum) est retiré de ce qu' il pourvoit»; puis celle d' un bref fragment du commentaire de la même proposition, et finalement la proposition $20 \mathrm{du}$ Liber de causis: Prima causa regit res, praeterquam commisceatur eis ${ }^{68}$.

2. Le corollaire suivant affirme : la bonté d'être, d' exister (essendi) des creatures dépend incontestablement du Premier. L' argumentation par l' autorité s' appuie sur celle d'Aristote (Métaphysique, l. II): primum in unoquoque genere est causa omnium quae sunt illius generis, et sur l' idée proclusienne de la chaîne des êtres (catena aurea ou argentea, aussi seyra). La première est comme un nœud indissoluble liant les secondes (les créés) au Premier, alors que la seconde chaîne unie les créatures entre elles ${ }^{69}$.

3. La discussion du corollaire suivant: après l' interruption de l'influence de la lumière de Dieu sur la figure des choses créées, ces choses ne retiendraient plus aucun bien, mais passeraient au néant. L'argumentation est longue à partir de l' autorité des Pères (Damascène et Grégoire de Nysse) ainsi que de Boèce (De hebdom. reg. 1a). Finalement nous avons: Proclus propositione 40: 'Omnia quae ab aliqua causa procedunt praecedunt, quae a se ipsis $\langle$ subsistunt $\rangle$ et habentia essentiam authupostaton, id est subsistentem $^{70}$. Suit immédiatement une ligne du commentaire de la même proposition: Quae a seipsis sunt et authupostaton, sunt seniora his quae ab alio (éd. Boese, p. 24, 9-11).

\section{Conclusion}

Il m'est difficile de conclure mon propos qui ne surprendra personne et qui, sur le plan doctrinal, n' apporte pas grande chose. En vérité, il est surprenant de constater que la tout première thèse théologique d'un grand maître parisien, celle qui impose et soutient la suite de la spéculation néoplatonicienne est, avec quelques légères modifications, empruntée à Proclus. Mais je n'ai lu et examiné que quelques colonnes de beaux manuscrits de Charlier, trop peu pour avoir une idée sur le contenu de son Commentaire des quatre livres des Sentences. Je me limiterai donc à quelques observations.

68 Mazar., 958, f. $3^{\text {va }}$ : Secunda pars quod bonitas divina non commisceatur rebus patet. Divinus refluxus in nullo (...). Cf. plus haut, texte à la note 44.

69 Mazar., 958, f. $3^{\text {va }}$ : Sequitur ultra quod bonitas essendi (...). Elementatio in pluribus locis, cf surtout prop. 125 .

$70 \quad$ Mazar., 958, f. $3^{\text {va }}$ : Sequitur ultra quod cessante influentia divini luminis. 
1. Les sources du Commentaire de Charlier sont déjà énumérées vers la fin de notre introduction. Il faut toutefois rappeler les principaux auteurs. Ce sont Aristote, Albert le Grand, Ps.-Denys l'Aréopagite, Proclus et le Liber de causis anonyme. Mais, lisant la Question collative et d' autres passages assez longs du Commentaire, je n' avait jamais l'opinion qu'Aristote participe aux débats, que les autres l'attaquent ou le défendent. Le Philosophe est là pour donner son opinion, un bout de phrase, une belle citation bien à propos, sortie des Auctoritates: la Bible des artistae. Celui qui mène le jeu s'appelle à présent Albert le Grand. Son héritage conserve tout: toutes les écoles de l'Antiquité, de Byzance, de Rome et de ses provinces. Il est donc sans cesse consulté et continue d'inspirer les universitaires. Grâce aux bons index de l'édition Coloniensis, il remplace avec succès les concordances manquantes: il a tout lu, tout noté et il est aujourd' hui plus intéressant que hier. Depuis que Saint Thomas les a unis au XIII siècle, on doit lire ensemble ces deux philosophes - Proclus et l'anonyme du De causis. Tous, ils continuent à être élèves du Maître Albert.

2. En préparant la présente contribution, j' ai gagné une ou deux convictions. La plus importante est celle-ci: avant de travailler sur Gille Charlier, j' aurais dû lire continuellement les grands œuvres d' Albert. J' aurais dû comparer «ma» question collative avec les grands écrits d'Albert, avec le De bono, la Summa theologiae, le Commentaire des Sentences, celui au De divinis nominibus. Aujourd'hui, je suis persuadé qu' un tel exercice m' aurait préparé à mieux comprendre Proclus.

3. Mes lectures parallèles, par exemple le bel ouvrage d'Alain de Libera intitulé Métaphysique et noétique Albert le Grand, m'ont appris ce qui se faisait et produit ailleurs, dans des écoles dominicaines allemandes, et qui restait très voisin des idées de Gilles Charlier, pourtant un chanoine cathédral. Telle est donc probablement la seule conviction positive de mon travail. Elle s' accompagne d'une interrogation supplémentaire: Gilles Charlier était-il en son temps le seul homme à Paris pour se battre de côté d' Albert et du néoplatonisme?

\section{Annexe: Mentions nominales de Proclus dans le premier Principium de Gilles Charlier (ms. Mazar. 958)}

1, fol. $2^{\text {rb }}$ : Pars $\langle$ conclusionis $\rangle$ quae dicit quod bonitas per essentiam arguitur per illud Matthei 19... Et Proclus in Elementatione theologica, propositione 121, dicit: Omne divinum essentiam habet bonitatem (Boese, p. 6o). 
2, fol. $2^{\text {va }}$ : Sequitur ultra quod $\langle$ Dei bonitas $\rangle$ tantum est una. Probatio. [...] Ideo Aristoteles $5^{\circ}$ Metaphysice: substantia cuiuslibet rei semel est, et Proclus propositione 5: Omnis multitudo secunda est ab uno, et 111: Ab uno et ad unum procedit quod est ex propria monade (Boese, p. 4 ; propos. 110 comm., p. 56, $12-$ 13).

3, fol. $2^{\mathrm{vb}}$ : Et Proclus propositione 8: Omnia qualitercumque participantia bono praecedit quod prime bonum 〈et quod $\rangle$ nichil aliud est quam bonum (Boese, p. 7).

4, fol. $3^{\text {va }}$ : Sequitur quod ista influentia $\langle$ boni $\rangle$ res salvat in esse praeterquam eis commisceatur. Probatur prima pars: unitate omnia salvantur et dispersio unumquodque facit exire a substantia, habet Proclus, propositione 11 (Boese, propos. 13, comm., p. 10, $5^{-6}$ ).

5, fol. $3^{\text {vb }}$ : Ideo Proclus propositione 122: Omne divinum exemptum est ab hiis quibus providet, et in commento eiusdem dicit: Manens enim in suo uniali omnia replet sua virtute. Ideo actor De causis propositione 20 : Prima causa, ait, regit res praeterquam commisceatur eis. (Boese, propos. 122 comm., p. 61, 5-6).

6, fol. $3^{\mathrm{vb}}$ : Ex quo patet quod cathena aurea et seyra, ut Proclus loquitur, est indissolubilis, quoniam illa est nexus secundorum ad primum, quamvis argentea, quae est habitudo creatorum ad invicem, est solubilis.

7 , fol. $3^{\text {vb }}$ Infero causa collationis habendae cum uno magistrorum meorum quod impossibile est creaturam fuisse ab aeterno probo sic primo ostensive... $2^{\circ}$, impossibile est terminos creationis simul esse... $3^{\circ}$, omne compositum est indigens partibus... sed omne citra Primum est compositum ex esse et quod est, Boethius libro De ebdomadibus... (fol. $4^{\mathrm{ra}}$ ) $4^{\mathrm{o}}$, processio causalis facit differentiam secundum creaturam: in creatore est eadem ei, ut dicit Damascenus, $2^{\circ}$ suarum Sentenciarum; bonitas (unitas?) vero ex necessitate est natura prior numero. Ergo prius $\langle$ est $\rangle$ ex creatura in creatore quam in seipsa. Igitur non potest fuisse producta ab aeterno. Ideo Proclus propositione 40: Omnia quae $\mathrm{ab}$ aliqua causa procedunt praecedunt quae a se ipsis $\langle$ subsistunt $\rangle$ et habentia essentiam antipostaton (lege authupostaton), id est (per se〉 subsistentem. Et ibidem in commento: Quae a se ipsis sunt et antipostata (lege authupostata) sunt, seniora $\langle$ sunt $\rangle$ hiis quae ab alio (Boese, comm. p. 24, 9-10). $5^{\circ}$, si creatura posset ab aeterno fuisse, sequerentur quatuor inconvenientia. Primum, quod creatura non surgeret ex nichilo per creationem... secundum, quod in aliqua creatura potentia nullo ordine praecederet actum... tertium, quod esset possibile esse multitudinem actu infinitam... quartum, quod creatura haberet potentiam infinitam et inextinguibilem et ne aequaretur creatori in duratione, ut arguebat beata Katherina philosophis ad hoc respondere nescientibus. Probatur argumento Procli commentis propositionum 84 et 86. Arguit enim sic. $\mathrm{Si}$ indeficiens est hypostasis, potentia secundum quam /est quod est et esse 
potest $\rangle$ est infinita. Si enim finita, deficeret aliquando et non sine defectu substantiae. Ergo semper ens est infinitum inextinguibile $\langle m\rangle$, habens potentiam, existentiam indeficientem et operationem imminorabilem (Boese, propos. 84, comm. p. 44, 1-2; propos. 86, comm. p. 44, 4-5);

8 , fol. $4^{\text {va }}$ : Ex quo sequitur quod ens et bonum ex aequo se habent secundum supposita, contra Platonem et Proclum, propositione 8, praeponente bonum enti in communitate. Et ibi patet falsitas opinionis Platonis... (Boese, p. 7).

\section{Bibliographie}

\section{Manuscrits}

Angers, Bibliothèque municipale, 204

Angers, Bibliothèque municipale, 205

Bruxelles, Bibliothèque royale, $11571-75$

Paris, Bibliothèque Mazarine, 958

Paris, Bibliothèque Mazarine, 959

Troyes, Bibliothèque municipale, 778

\section{Sources primaires}

***, Il Libro di ventiquattro filosofi, éd. P. Lucentini, Milano, Adelphi, 1999; ***, Le Livre des XXIV Philosophes, trad., éd. et notes par F. Hudry, Grenoble, Jérôme Million, 1989 .

Auctoritates Aristotelis, Un florilège médiéval. Étude historique et édition critique, éd. J. Hamesse, Louvain / Paris, Publications Universitaires / B. Nauwelaerts, 1974.

Albert le Grand, Metaphysica, éd. B. Geyer, Opera omnia, éd. Colon. t. Xvı.1, Münster, Aschendorff, 1960.

Albert le Grand, De causis et processu universitatis a prima causa, éd. W. Fauser, Opera omnia, éd. Colon. t. XVII.2, Münster, Aschendorff, 1993.

Albert le Grand, Super Dionysium De divinis nominibus, éd. P. Simon, Opera omnia, éd. Colon. XxxviI.1\&2, Münster, Aschendorff, 1972-1978.

Aristote, De sophisticis elenchis, transl. Boethii, éd. B.G. Dod, Aristoteles Latinus VI.1-3, Leiden / Bruxelles, Brill / Desclée de Brouwer, 1975.

Aristote, Metaphysica, transl. Anonyma, éd. G. Vuillemin-Dim, Aristoteles Latinus Xxv.2, Leiden, Brill, 1976.

Aristote, Ethica Nicomachea, transl. Roberti Grosseteste Lincolniensis, éd. R.A. Gauthier, Aristoteles Latinus Xxvi 1-2, fasc. 3, Leiden / Bruxelles, Brill / Desclée de Brouwer, 1972.

Augustin, De Trinitate, trad., notes M. Mellet, Th. Camelot, introd. E. Hendrikx, Bibliothèque Augustinienne, Oeuvres de saint Augustin 15, Paris, Desclée de Brouwer, 1955. 
Averroès, In Metaphysica, Venezia, apud Junctas, 1562-1574 [repr. Frankfurt am Main, Minerva, 1962], vol. viII.

Avicenne [Avicenna latinus], Liber de philosophia prima sive scientia divina, éd. S. Van Riet, Louvain / Leiden, Peeters / Brill, 1980.

Boèce, De consolatione philosophiae; Opuscula theologica, éd. C. Moreschini, Munich / Leipzig, Teubner, 2000 [ $2^{\mathrm{e}}$ éd. 2005 ]

Boèce, Quomodo substantiae, dans Boethius, De consolatione philosophiae; Opuscula theologica.

Boèce, De sancta trinitate, dans Boethius, De consolatione philosophiae; Opuscula theologica.

Denys l'Aréopagite, Dionysiaca - Recueil donnant l'ensemble des traductions latines des œuvres attribuées au Denys de l'Aréopagite, I-II, éd. Ph. Chevallier, Brugge, Desclée de Brouwer, 1937 [Faksimile-Neudruck in vier Bänden mit einem Nachwort von Martin Bauer, Stuttgart-Bad Cannstatt, Frommann-Holzboog, 1989].

Heimeric de Campo, Compendium divinorum, dans Korolec 1967.

Heimeric de Campo, Opera selecta, 1, éd. R. Imbach, P. Ladner, Fribourg, Universitätsverlag, 2001.

Heymericus de Campo, Tractatus de naturali veritatis catholice analesy, dans Heymericus de Campo, Opera selecta.

Jean Gerson, OEuvres complètes, vol. I, Paris / Tournai, Desclée de Brouwer, 196o.

Jean Gerson, Épîtres, dans CEuvres complètes, éd. P. Glorieux, vol. II : L'OEuvre épistolaire, Paris / Tournai, Desclée de Brouwer, 1960.

Jean Gerson, Duae lectiones contra vanam curiositatem, dans OEuvres complètes, éd. P. Glorieux, vol. III: L'OEuvre magistrale, Paris / Tournai, Desclée de Brouwer, 1962.

Michel d' Éphèse, The Greek Commentaries on the Nicomachean Ethics of Aristotle in the Latin Translation of Robert Grosseteste Bishop of Lincoln (†1253), vol. III: The Anonymous Commentator on Book VII, Aspasius on Book VIII and Michael of Ephesus on Books IX and X, éd. H.P.F. Mercken, Leuven, University Press, 1991.

Proclus, Elementatio theologica translata a Guillelmo de Morbecca, éd. H. Boese. Leuven, Leuven University Press, 1987; Proclus, Éléments de théologie, trad. introd. et notes par J. Trouillard, Paris, Aubier, Éditions Montaigne, 1965.

Thomas d' Aquin, Super Librum de causis expositio, éd. H.D. Saffrey, Fribourg / Louvain, Société Philosophique / Éditions E. Nauwelaerts, 1954.

Thomas d' Aquin, In duodecim libros Metaphysicorum Aristotelis expositio, éd. M.R. Cathala, R.M. Spiazzi ( $2^{\mathrm{a}}$ éd.) Torino / Roma, Marietti, 1971. 


\section{Sources secondaires}

Calma, D. (2016) (éd.), Neoplatonism in the Middle Ages. Vol. I: New Commentaries on Liber de causis (ca.1250-1350); Vol. II: New Commentaries on Liber de causis and Elementatio theologica (ca. 1350-1500), Turnhout, Brepols.

Calma, D., Imbach, R. (2008), «A Fifteenth-Century Metaphysical Treatise: Preliminary Remarks on the Colliget principiorum of Heymericus de Campo», dans Przeglad Tomistyczny 14, p. 225-276.

Calma, D., Imbach, R. (2013), «Heymeric de Campo, auteur d'un traité de métaphysique. Étude et édition partielle du Colliget principiorum », dans Archives d'Histoire Doctrinale et Littéraire du Moyen Âge 80, p. 277-423.

Chappuis, M. (1993), Le Traité de Pierre d'Ailly sur la Consolation de Boèce, Amsterdam / Philadelphia, Grüner.

Doucet, V. (1939), «Magister Aegidius Carlerii (†1472) eiusque quaestio de immaculata conceptione b. Mariae Virginis », dans Antonianum v, p. 405-442.

Glorieux, P. (196oa), Introduction générale, dans Jean Gerson, CEuvres complètes, vol. I.

Glorieux, P. (196ob), Essai biographique, dans Jean Gerson, QEuvres complètes, vol. I.

Glorieux, P. (1969) «L'enseignement au Moyen Âge. Techniques et méthodes en usage à la Faculté de Théologie de Paris, au XIII ${ }^{\mathrm{e}}$ siècle», dans Archives d'Histoire Doctrinale et Littéraire du Moyen Âge 35, p. 65-186.

Gorochov, N. (1997), Le collège de Navarre de sa fondation (1305) au début du XV siècle (1418). Histoire de l'institution, de sa vie intellectuelle et de son recrutement, Paris, Honoré Champion, p. 6o9-610.

Hadot, P. (1999), «Conversio», dans Id., Plotin, Porphyre. Ėtudes néoplatoniciennes, Paris, Les Belles Lettres, p. 37-42.

Hoenen, M.J.F.M. (2002), «Academic Theology in the Fifteen Century, The Sentences Commentary of Heymericus de Campo», dans P.J.J.M. Bakker, E. Faye, C. Grellard (éds), Chemins de la pensée médiévale, Turnhout, Brepols, p. 513-559.

Kaluza, Z. (1986), «Le De universali reali de Jean de Maisonneuve et les Epicuri litterales », dans Freiburger Zeitschrift für Philosophie und Theologie 33, p. 469-516.

Kaluza, Z. (1987), «La nature des écrits de Jean de Ripa», dans Traditio 43, p. 257298.

Kaluza, Z. (1988), Les querelles doctrinales à Paris. Nominalistes et réalistes aux confins du XIV $v^{e}$ et du $X v^{e}$ siècles, Bergamo, Lubrina.

Kaluza, Z. (1992), «La voix créatrice de Dieu. Remarques sur l'Alphabetum de Heimeric de Campo», dans H.J. Westra (éd.), From Athens to Chartres. Neoplatonism and Medieval Thought. Studies in Honour of Edouard Jeauneau, Leiden, Brill, p. 439468.

Kaluza, Z. (1995), «Les débuts de l'albertisme tardif (Paris et Cologne)», dans M.J.F.M. Hoenen, A. de Libera (éds), Albertus Magnus und der Albertismus. Deutsche philosophische Kultur des Mittelalters, Leiden, Brill, p. 207-295. 
Kaluza, Z. (1998), «Gerson critique d'Albert le Grand», dans Freiburger Zeitschrift für Philosophie und Théologie 45.1-2, p. 169-205.

Kaluza, Z. (2011) «Pater, paternitas», dans I. Atucha, D. Calma, C. König-Pralong, I. Zavattero (éds), Mots médiévaux offerts à Ruedi Imbach, Porto, FIDEM, p. 527-534.

Kaluza, Z. (2015), «Supremum vale: le discours de clôture du Commentaire des Sentences de Gilles Charlier», dans Przeglad Tomistyczny xxI, p. 327-345.

Korolec, J.B. (1967-1968), «Compendium divinorum Heimeryka de Campo w rkp. 695. Studia nad dziejami albertyzmu kolońskiego », dans Studia mediewistyczne 8, p. 1975 et 9, p. 3-9o.

Libera, A. de (1993), La philosophie médiévale, Paris, P.U.F.

Libera, A. de (1999) L'Art des généralités. Théories de l'abstraction, Paris, Aubier.

Libera, A. de (2005), Métaphysique et noétique d'Albert le Grand, Paris, Vrin.

Meersseman, G. (1933), Geschichte des Albertismus. Heft I: Die Pariser Anfänge des Kölner Albertismus, Paris, R. Haoula.

Meliadò, M., Negri, S. (2011), « Neues zum Pariser Albertismus des frühen 15. Jahr hunderts. Der Magister Lambertus de Monte und die Handschrift Brussel, Koniklijke Bibliotheek, ms. 760 », dans Bulletin de philosophie médiévale 53, p. 349-384.

Omont, H. (1919), «Inventaire des livres de Jean Courtecuisse, évêque de Paris et de Genève», dans Bibliothèque de l'École des chartes 80, p. 109-120.

Ouy, G. (1989), «Les premiers humanistes et leurs livres», dans A. Vernet (éd.), Histoire des bibliothèques françaises. I - Les bibliothèques médiévales $d u V^{e}$ siècle à 1530, Paris, Édition du Cercle de la Librairie, p. 365-381 + Annexes de F. Bérard, p. $382-385$; dans l'édition richement illustrée Paris, Promodis, 1989, p. 267-284 (sans Annexes).

Sullivan, T. (2011), Parisian Licentiates in Theology, A.D. 1373-1500: A Biographical Register. Vol. 2: The Secular Clergy. Leiden, Brill.

Swieżawski, S. (1963), «Les reflets des problèmes métaphysiques au Moyen Âge tardif dans le Commentaire des Sentences de Gilles Charlier (†1472)», dans P. Wilpert, W.P. Eckert (éds), Die Metaphysik im Mittelalter. Ihr Ursprung und ihre Bedeutung: Vorträge des II. Internationalen Kongresses für mittelalterliche Philosophie, Köln 31.8.6.9.1961 (Miscellanea mediaevalia 2), p. 732-742.

Swieżawski, S. (1961), «Note sur le Commentaire des Sentences de Gilles Charlier», dans Mediaevalia Philosophica Polonorum X, p. 77-86.

Toussaint, J. (1949), «Gilles», dans Dictionnaire d'Histoire et de Géographie Écclésiastiques, t. XI, Paris, Letouzey et Ané, col. 1046-1050.

Vignaux, P. (1962), «L'Être comme perfection chez François de Meyronnes», dans Études d'histoire littéraire et doctrinale, Montréal, Institut d'études médiévales, p. $259-318$.

Vignaux, P. (1976), «L'Être comme perfection chez François de Meyronnes», dans Id., De saint Anselme à Luther, Paris, Vrin, p. 253-312. 
Zahnd, U. (2016), «Der Dank an die Meister. Anmerkungen zu einiger gratiarum actiones spätmittelalterlicher Sentenzenlesungen», dans A. Speer, Th. Jeschke (éds), Schüler und Meister (Miscellanea Mediaevalia 39), Berlin, de Gruyter, p. 81-105. 